\title{
Prevalence of Overweight/Obesity and Its Association with Socioeconomic Status in Ethiopia: Systematic Review and Meta-Analysis
}

\author{
Tona Zema Diddana* \\ School of Nutrition, Food Science and Technology, Hawassa University, Hawassa, Ethiopia
}

*Corresponding author: Tona Zema Diddana, School of Nutrition, Food Science and Technology, Hawassa University, Hawassa, Ethiopia

\begin{abstract}
Background: The prevalence of overweight and obesity had considerably increased in developing countries including Ethiopia. In Ethiopia, there is a large variation in prevalence across different regions. There is also inconsistency in the relationship of socioeconomic status (SES) and overweight/obesity across regions. These inconsistencies could make policymakers difficult to make a decision and designing appropriate intervention strategies. Hence, this systematic review and meta-analysis was aimed to generate a pooled prevalence of overweight/obesity and its association with socioeconomic status in Ethiopia, 2020.
\end{abstract}

Methods: The articles used in this study identified through the electronic search of reputable databases (Google Scholar, EMBASE, Science Direct, PubMed Cochrane library) and hand search of reference lists of previous prevalence studies. Overall, 27 studies were selected based on specified inclusion and exclusion criteria. Data were extracted using a standardized and pre-tested data extraction checklist. The SPSS software program version 20 was used for pooled prevalence analysis while Review Manager 5.3 was used to estimate the pooled effect size of SES and obesity/ overweight. The random effect model was used in the meta-analysis and Mantel-Haenszel model was used to calculate odds ratios and their $95 \%$ confidence interval.

Result: The pooled prevalence of overweight/obesity among the study population (children, adolescents, and adults) was $13.04 \%(\mathrm{Cl}: 12.64 \%, 13.43 \%)$ in Ethiopia. There is a highly positive significant association between high SES/income and overweight/obesity among study subjects (children, adolescents, and adults) in Ethiopia. Accordingly, study populations from higher SES/income groups were 2.35 times higher $(\mathrm{OR}=2.35,95 \% \mathrm{Cl}: 1.89$, 2.92) times more likely to be overweight/obese compared to other low SES/income groups.
Conclusion: The result revealed that the pooled prevalence of overweight/obesity among children, adolescents, and adults is considerably high, and high SES/income contributes to the risk of overweight/obesity in different population groups in Ethiopia. Therefore, the government of Ethiopia and other concerned bodies should emphasize the prevention and control of overweight/obesity. Intervention strategies and policies with special emphasis on wealthy households should be designed and implemented.

\section{Keywords}

Overweight, Obesity, Socio-economic status, Ethiopia

\begin{abstract}
Abbreviations
Cl: Confidence Interval; EDHS: Ethiopian Demographic and Health Survey; EMBASE: Excerpta Medica data BASE; FAO: Food and Agricultural Organization; IFAD: International Fund for Agricultural Development; O: Obesity; OR: Odds Ratio; OW: Overweight; PRISMA: Preferred Reporting Items for Systematic Reviews and Meta-Analyses; SES: Socio-economic Status, UNICEF: United Nations International Children's Emergency Fund; WFP: World Food Program
\end{abstract}

\section{Background}

World health organization defined overweight and obesity as an excessive accumulation of fat that may impair health [1]. The center for disease control and prevention stated that nearly $30 \%$ of the world population is estimated to be either overweight or obese in 2015, of which two-thirds are living in developing countries [2]. About 38.3 million under five-children was obese [3]. About 2.01 billion adults are overweight

Citation: Diddana TZ (2021) Prevalence of Overweight/Obesity and Its Association with Socioeconomic Status in Ethiopia: Systematic Review and Meta-Analysis. J Obes Weight-loss Medic 7:042. doi. org/10.23937/2572-4010.1510042

Accepted: December 03, 2021: Published: December 05, 2021

Copyright: (C) 2021 Diddana TZ. This is an open-access article distributed under the terms of the Creative Commons Attribution License, which permits unrestricted use, distribution, and reproduction in any medium, provided the original author and source are credited. 
in 2016 of which 678 million are obese worldwide. Data also implies that the prevalence of overweight and obesity among adults (> 18 years) showed significant increment such that it was raised from 35.7-38.9\% and 11.2-13.1\%, respectively, from 2010 to 2016 [4]. According to the FAO, IFAD, UNICEF, WFP, and WHO 2019 overweight and obesity continue to increase in all regions. Accordingly, in 2018, nearly 40 million children under five were overweight. In 2016, 131 million children 5-9 years-old, 207 million adolescents, and 2 billion adults were overweight. About a third of overweight adolescents and adults, and 44 percent of overweight children aged 5-9 were obese [5].

The prevalence of overweight/obesity has increased in many developing and low-income countries including Ethiopia. As per available literature from local studies in Ethiopia, the magnitude of overweight/obesity is considerably high and rising in all age groups. According to EDHS 2016, the proportion of women who were overweight or obese has increased from 3\% in 2000 to $8 \%$ in 2016 [6]. Furthermore, based on the Ethiopian Demographic and Health Survey report, the prevalence of obesity and or overweight increased from 2-2.8\%, 6-8\%and $2-3 \%$ among under-five children, $15-49$ yearsold women, and 15-49 years-old men, respectively, between 2011 and 2016 [6,7]. The UNICEF 2017 annual report also stated that there is an overall increment of the prevalence of overweight among children from 1.7 to $3.6 \%$ in Ethiopia [8].

Overweight and obesity have a negative impact. Overweight and obesity have negative impacts. Pieces of evidence illustrate that obesity is contributing to 4 million deaths globally and is increasing the risk of morbidity for people in all age groups [5]. Overweight or obese population groups are at a higher risk of developing serious health problems such as type 2 diabetes, high blood pressure, asthma, and other respiratory problems, sleep disorders, liver disease, low self-esteem, depression, and social isolation [9]. Obesity has an important contribution to cancer, osteoarthritis, work disability, and sleep apnea and it has a more pronounced impact on morbidity than on mortality $[10,11]$.

In Ethiopia, it has been documented that there is a significant variation in the prevalence of overweight/ obesity and its association with socio-economic status (SES) across different regions of the country. Local survey recognized that the prevalence obesity varied from 2.8-13.8\% [6,12], 5.1-20.5\% [13,14], and 8.3$28.5 \%[15,16]$ among children, adolescents and adults in Ethiopia, respectively. On the other hand, there is an inconsistency in the relationship between SES and overweight/obesity across different age groups in the country. Some studies reported that higher SES has no significant association with obesity/overweight [12,1722]. Other scholars described that there was a negative relationship [13,23], while other studies indicated a positive significant association [24-27].

These disagreements could make policymakers difficult on decision making of designing intervention strategies, implementation, and evaluation. Hence, a better understanding of the prevalence of overweight/ obesity across different age groups (children, adolescents, and adults) across different regional groups in particular and country in general will aid decision-making about the best way to implement and evaluate a range of policies and strategies at a national level. On the other hand, understanding the association of socioeconomic status with overweight/obesity also benefits the government and health care provider to provided and focus appropriate nutrition education and counseling for high income or wealthy households. Therefore, this systematic review and meta-analysis was aimed to generate a pooled prevalence of overweight and/or obesity and its association with socioeconomic status in Ethiopia, 2020.

\section{Methods}

\section{Study design, study period and literature search approach}

This systematic review and meta-analysis was directed by PRISMA (Preferred Reporting Items for Systematic Reviews and Meta-Analyses) [28]. The literature search was conducted from February to April 2020. All articles until April 30, 2020 were included in this review study. An electronic search of reputable databases (Google Scholar, PubMed, Science Direct, Cochrane library, EMBASE,) was used to identify appropriate articles for this study. Moreover, a hand search of reference lists of previous prevalence studies to find more related and relevant articles was conducted. The keywords used for searching relevant literature were: (i) Population (children, schoolchildren, school-aged, childhood, adolescent, and adults); (ii) Outcome (body composition, overweight, obesity, body weight, weight status, body mass index, anthropometric status (iii) Study design (prevalence, cross-sectional and case-control); and (iv) Specific study area (Ethiopia and regions in Ethiopia). The English language was used to search for articles. Review Manager 5.3 was used to manage references.

\section{Inclusion criteria}

The articles were included based on the following criteria: (1) Study population: research articles conducted among children, adolescents and adults (2) All prevalence and other cross-sectional studies; and case-control studies; (3) A study conducted and published in the English language were considered. In addition, prevalence studies conducted on children, adolescents and adults were included regardless year of study. 


\section{Exclusion criteria}

Articles with methodological problems, other than cross-sectional study and did not contain full information and articles that did not contain socio-economic status result were excluded.

\section{Data abstraction and critical appraisal of the studies}

Data were extracted by preparing a standardized checklist in Ms-excel sheet version 2013. The extracted data from included articles are the primary author of the article, specific area of the study conducted in Ethiopia, study design, diagnostic information, year of the publication, calculated sample size, response rate, and outcome measures (prevalence of overweight/ obesity). The Newcastle-Ottawa quality assessment tool scale was used to appraise the qualities of the studies. The qualities of the studies were assessed by using the following indicators; those with medium (fulfilling $50 \%$ of quality assessment criteria) or high quality ( $\geq 6$ out of 10 scales) were considered for inclusion in the metaanalysis.

\section{Identification of eligible studies}

The procedures followed for identification and selection of relevant studies is indicated in Figure 1. Totally, 602 articles were searched from electronic data base. Duplicate record $(n=328)$ were removed from total searched study. One hundred thirty four $(n=134)$ were excluded by title and $(n=66)$ were by title and abstract. One hundred forty $(n=140)$ were excluded since they were not relevant for this meta-analysis in terms of outcome the study is interested. Eighty three ( $n=74$ ) full text articles were assessed for eligibility based on the pre-set eligibility criteria of which 47 were excluded as per exclusion criteria. Finally, 27 studies were fulfilled inclusion criteria were included in analysis.

\section{Operationalization of the outcomes of the review}

The number of overweight/obese adolescents and adults were the primary outcome of this review and the secondary outcome was socio-economic status defined by wealth index or highest income status. The prevalence of overweight and obesity was calculated by dividing the number of overweight/obese adolescents and/or adults to the total number of adolescents and/or adults included in this study multiplied by 100 (equation 1). The strength and direction of the association between overweight/obesity and socioeconomic status were quantified by the odds ratio. The participants were categorized into high either high socio-economic status group or low socio-economic status group for the purpose of analysis. The high socio-economic status category in this systematic review and meta-analysis study is operationally defined as those categorized highest quantiles or wealthiest or highest monthly income group in original study The low socio-economic

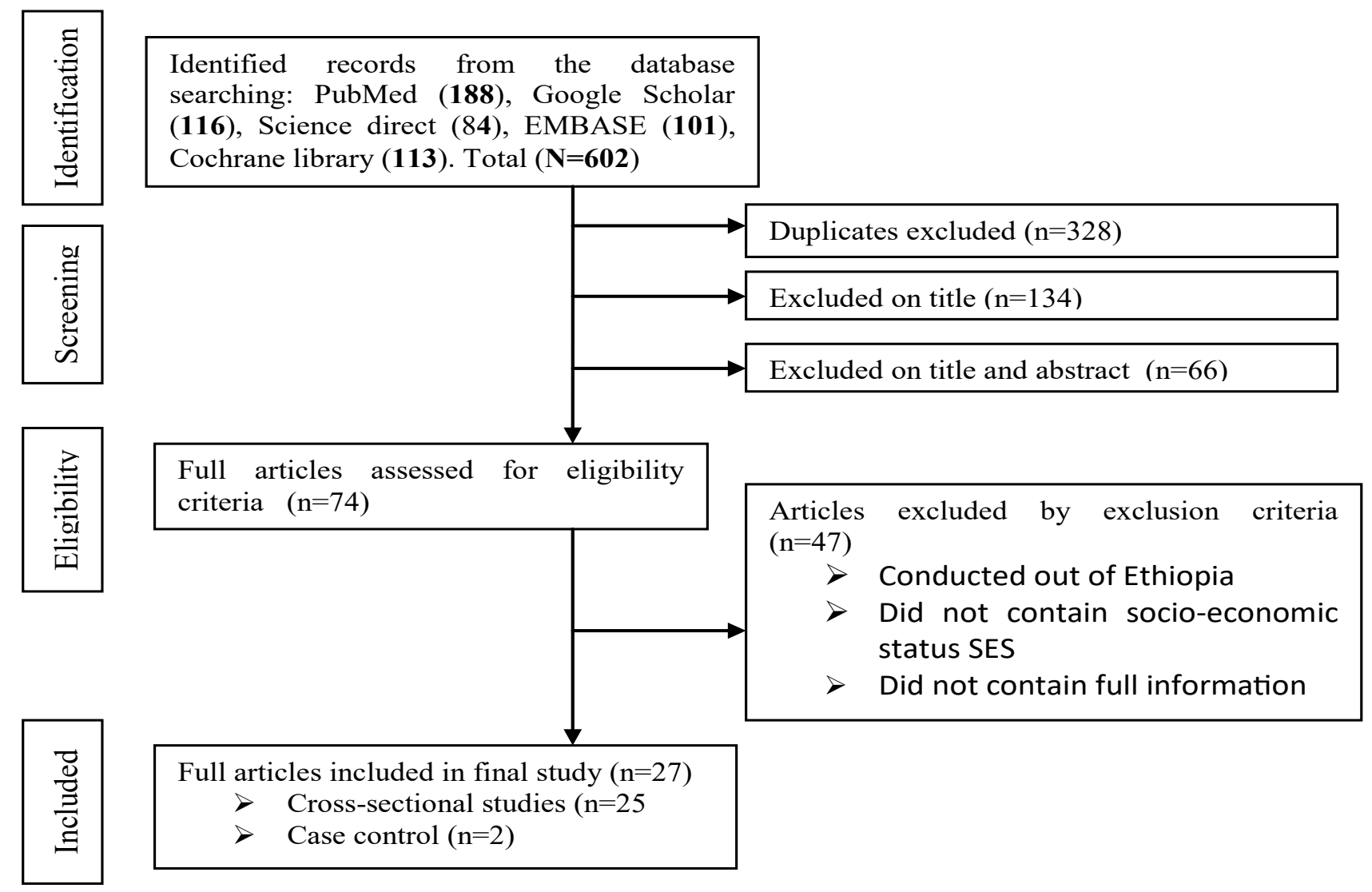

Figure 1: Flow chart diagram describing selection of studies for the systematic review and meta-analysis of magnitude of overweight/obesity and its association with socio-economic status (SES) in Ethiopia, 2020 G.C. 
status groups in this study were those medium to low quantiles or having lower monthly income or medium and poor in original study. (Wealthiest/highest income $=1$; and the rest wealth/income category $=0$ ).

Magnitude of overweight obesity $=\frac{\text { Number of overweight } / \text { obese adolescents and / adults }}{\text { Total number of participants included this study }} \times 100$

\section{Data analysis/synthesis of results}

The data for the prevalence of overweight/obesity was analyzed by using the SPSS statistical software program version 20. The association of outcome variables with socio-economic status (SES) was pooled using Review Manager 5.3 (RevMan 5.3) [28]. The random effect model was used in meta-analysis and Mantel-Haenszel model was used to calculate odds ratios and their 95\% confidence interval. The original studies were summarized and presented by using a table and the forest plot. The potential heterogeneity among the reported prevalence of the studies was explored using $I^{2}$ test and Cochrane $Q$ statistics [29]. Since the test statistics revealed that there was considerable heterogeneity $\left(\mathrm{I}^{2}=84 \%, \mathrm{P} \leq 0.001\right)$ among the studies, a random-effects model was used to estimate the Der Simonian and Laird's pooled effect [30]. A univariate meta-regression model analysis conducted to identify the likely sources of variation. Year of publication and sample size were used as input variables in univariate meta-regression. Both of them did not show any significant variation (Table 1). Any publication bias was examined by Egger's weighted correlation and Begg's regression intercept tests at 5\% significant level [31]. The test results showed there is a significant publication bias ( $p \leq 0.001$ ). The final effect size was determined by applying Duval and Tweedie's Trim and Fill analysis in the Random-effects model.

\section{Result}

\section{Description of included studies}

Overall, 602 relevant papers identified through literature search strategy. Three hundred twenty eight (328) articles were identified as duplicates and 274 were retrieved for evaluation. Following study objectives, exclusion and inclusion criteria, 134 were excluded by title, 66 were excluded from abstract and title review. The full text of 74 papers was retrieved further detailed evaluation and methodological quality. Accordingly, 47 were excluded by appraisal and suboptimal quality. Finally, 27 articles were included in this systematic review and meta-analysis.

Table 1: Univariate meta-regression for related factors with the heterogeneity of overweight/obesity prevalence among study subjects in Ethiopia.

\begin{tabular}{|l|l|l|}
\hline Variables & Coefficient $(\boldsymbol{\beta})$ & p-value \\
\hline Year of publication of the studies & 0.46 & 0.082 \\
\hline Sample size of the studies & -0.23 & 0.362 \\
\hline
\end{tabular}

More than $96 \%$ of included studies in meta-analysis were cross-sectional in design and the remaining was case-control. All studies were conducted in different regions of Ethiopia. The minimum and maximum sample sizes included in original studies were 206 and 5465. Twenty five (25) articles from cross-sectional were used for pooled prevalence analysis and twenty seven (27) articles (cross-sectional studies $=25$ and case-control studies $=2$ ) were used for meta-analysis to estimate pooled effect size.

\section{Prevalence of overweight/obesity in Ethiopia}

The pooled and individual prevalence of obesity and/or overweight reported by 25 studies are described in Table 2. Overall, 27,818 subjects (children $=11,488$, adolescents $=10,738$, adults $=5,594$ ) subjects were included to estimate pooled prevalence of overweight/ obesity. Nine hindered ninety six (996) subjects did not include determining prevalence since they are from case-control study. The pooled prevalence of overweight/obesity was $13.04 \%$ (Cl: $12.64 \%, 13.43 \%)$. The subgroups or separate population group analysis revealed that the pooled prevalence of overweight/ obesity among children, adolescents and adults was $12.59 \%(\mathrm{Cl}: 11.98 \%, 13.19 \%), 11.39 \%$ (Cl: $10.79 \%$, $11.99 \%)$ and $17.13 \%(\mathrm{Cl}: 16.14 \%, 18.11 \%)$ respectively.

\section{Association of socioeconomic status with overweight/obesity}

The pooled effect size of socio-economic status and obesity/overweight is depicted in the forest plot (Figure 2). In meta-analysis, 27 studies were included. Overall, 28,814 subjects were retrieved in metaanalysis to estimate pooled effect size, and stratified as high SES/Income groups and low SES/income groups. Accordingly, 9327 subjects were from high SES/income groups and 19487 subjects were from low SES/income groups. Of these high SES/income groups, $17.65 \%$ were either overweight/obese. Among 28 included articles in a meta-analysis, five studies [20,21,32-34] did not show significant association while the rest 22 studies [12-14,16,17,19,22-27,32,35-43] showed a positive association between high SES/income and obesity/ overweight. The pooled effect size revealed that the likelihood of being overweight/obese is 2.35 times higher $(A O R=1.89,95 \% \mathrm{Cl}: 1.75,2.04)$ among high SES or high income groups compared to the low SES or income groups, heterogeneity test: Tau $=0.25, \mathrm{I}^{2}=84 \%$, $\mathrm{df}=26, \mathrm{p} \leq 0.001$. The chi-square test $\left(\mathrm{Chi}^{2}=160.90\right.$, $\mathrm{df}=26)$ indicated that there is significant $(P<0.001)$ variation in set of studies. The test for overall effect $(Z$ $=7.67$ ) showed statistically significance at conventional levels $(P \leq 0.001)$.

The forest plot for the separate population group analysis (children, adolescents and adults) is depicted in Figures 3. The pooled odds ratio for separate population groups indicated the risk of overweight/obesity was 
Table 2: Summary of characteristics of the studies reported on prevalence of overweight/obesity among children, adolescents and adults in Ethiopia in this current systematic review and meta-analysis, 2020.

\begin{tabular}{|c|c|c|c|c|c|}
\hline $\mathbf{S} / \mathbf{N}$ & $\begin{array}{l}\text { Authors and year of } \\
\text { publication }\end{array}$ & Study area & Sample size (n) & Quality of score & $\%(95 \% \mathrm{Cl})$ \\
\hline & Mekonnen, et al., 2018 & Bahirdar & 616 & 7 & $11.85(9.30,14.40)$ \\
\hline & Sorrie, et al., 2017 & Gondar & 500 & 7 & $13.80(10.78,16.82)$ \\
\hline & Tsedeke and Tefera, 2014 & Hawassa & 358 & 5 & $10.61(7.42,13.81)$ \\
\hline & Birhanu, et al., 2019 & Diredawa & 590 & 6 & $11.53(8.95,14.10)$ \\
\hline & Dessalegn, et al., 2017 & South Ethiopia & 600 & 7 & $11.17(8.65,13.69)$ \\
\hline & Berhane, et al., 2020 & Addis Ababa & 5822 & 9 & $11.41(10.59,12.22)$ \\
\hline & Solomon, et al., 2020 & Ethiopia & 3602 & 9 & $14.83(13.66,15.99)$ \\
\hline & Askal, et al., 2015 & Addis Ababa & 828 & 8 & $9.78(7.76,11.81)$ \\
\hline & Dessalew, et al., 2017 & Diredawa & 448 & 7 & $20.54(16.79,24.28)$ \\
\hline & Dessalegn, et al., 2018 & Addis Ababa & 390 & 7 & $18.21(14.38,22.03)$ \\
\hline & Kalkidan, et al., 2017 & Jimma Zone & 550 & 7 & $7.09(4.95,9.24)$ \\
\hline & Mulugeta, et al., 2016 & Addis Ababa & 446 & 7 & $11.25(11.91,18.58)$ \\
\hline & Tesfalem, et al., 2013 & Hawassa & 554 & 7 & $12.82(10.03,15.60)$ \\
\hline & Tsedey, et al., 2018 & Addis Ababa & 1276 & 9 & $17.01(14.94,19.07)$ \\
\hline & Zelalem, et al., 2015 & Bahirdar & 407 & 7 & $16.46(12.86,20.06)$ \\
\hline & Emana, et al., 2014 & Addis Ababa & 800 & 8 & $9.38(7.36,11.39)$ \\
\hline & Bogale and Zewale, 2019 & Hawassa City & 445 & 7 & $29.21(24.99,33.44)$ \\
\hline & Darebo, et al., 2019 & Hawassa & 524 & 7 & $28.24(24.39,32.10)$ \\
\hline & Gutema, et al., 2020 & Arbaminch & 3346 & 9 & $10.79(9.74,11.84)$ \\
\hline & Samuel, et al., 2019 & Dessie & 673 & 8 & $28.68(25.26,32.09)$ \\
\hline & Nega and Beyene, 2019 & Southern Ethiopia & 206 & 6 & $24.76(18.86,30.65)$ \\
\hline & Yosef et al., 2020 & Ethiopia & 400 & 6 & $31.25(26.71,35.79)$ \\
\hline & Mekonnen and Bogale, 2017 & Ethiopia & 2938 & 8 & $3.44(2.78,4.10)$ \\
\hline & Girmay and Hassen, 2014 & Addis Ababa & 950 & 7 & $14.95(12.68,17.21)$ \\
\hline & Nurezeman, et al., 2017 & Jimma & 549 & 7 & $19.49(16.18,22.80)$ \\
\hline \multicolumn{3}{|c|}{ Total } & $28124(98.38 \%)$ & Average (7.28) & $13.04(12.64,13.43)$ \\
\hline
\end{tabular}

${ }^{*}=$ the value is pooled prevalence.

2.90 times higher $(\mathrm{AOR}=2.90,95 \% \mathrm{Cl}=1.94,4.36), 2.36$ times higher $(\mathrm{AOR}=2.36,95 \% \mathrm{Cl}=1.52,3.65)$ and $87 \%$ higher $(A O R=1.87,95 \% \mathrm{Cl}=1.46,2.41)$ among children, adolescents and adults from high SES/income groups compared to low SES/income groups respectively.

\section{Discussion}

Overweight and/or obese during childhood and adolescence is associated with adverse health consequences throughout the life span leading to greater risk and earlier onset of chronic disorders such as type 2 diabetes, and adverse psychosocial consequences and lowers educational attainment $[44,45]$. Overweight and/or obesity in adults also associated with numerous comorbidities such as cardiovascular diseases, high blood cholesterol, and sleep apnea [46].

In Ethiopia, prevention and control of overweight/ obesity did receive lower attention compared to undernutrition despite it is an emerging problem leading to a double burden. The low attention in combination with limited information might increase the epidemiology of overweight/obesity among children, adolescents' adults leading to non-communicable diseases in future life. A few cross-sectional studies conducted in Ethiopia explored considerable variation in prevalence and associated risk factors of overweight/ obesity among children, adolescents, and adults across different regions. A few studies conducted showed the inconsistent result of overweight/obesity and its association with socio-economic status (SES) or wealth quantiles. This might contribute to policy and intervention strategies gaps in the country. Therefore, this systematic review and meta-analysis was aimed to explore the pooled prevalence of overweight/obesity and its association with socioeconomic status among children, adolescents and adults in Ethiopia.

The result of a pooled analysis of this study revealed that the prevalence of overweight/obesity was $13.04 \%$ in Ethiopia. This finding is lower than the result of the pooled prevalence of overweight/obesity (15.9\%) from the Demographic Health Survey (DHS) analysis of 32 Sub-Saharan African countries [47]. On the other hand, 


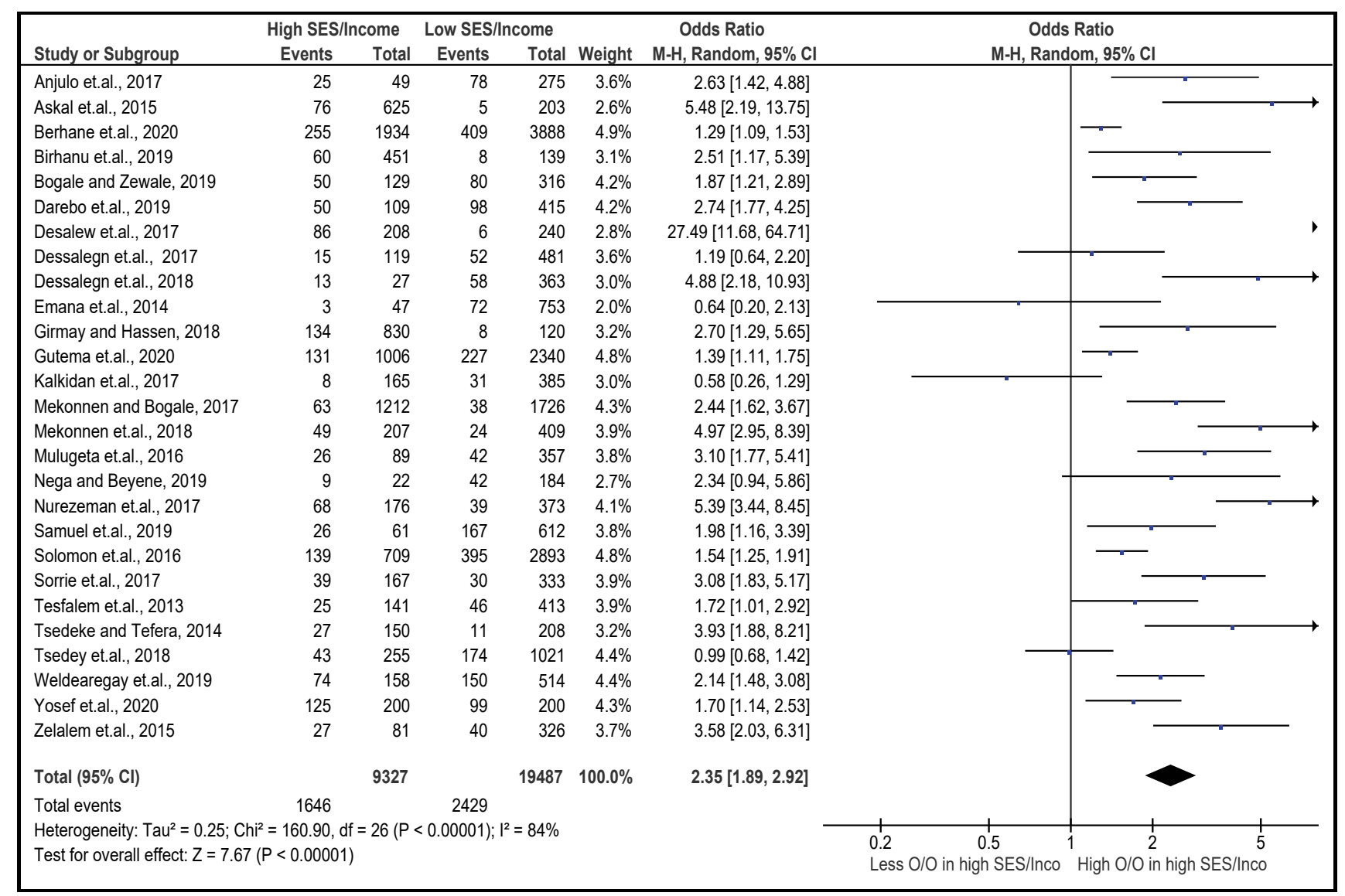

Figure 2: Forest plot Socio-economic status (SES) versus overweight/obesity $(\mathrm{O} / \mathrm{O})$ among children, adolescents and adults in Ethiopia, 2020.

it is comparable with another meta-analysis result conducted in china where the prevalence of overweight/ obesity among children and adolescents was $13.1 \%$ by the year 2010 in china [48]. Another study from United States National Nutrition and Health Examination survey 2015-2016 indicated a higher prevalence of overweight (16.6\%) and obesity (18.5\%) among children adults compared the current study.

The subgroup analysis revealed that the prevalence of pooled overweight/obesity among children, adolescents and adults was $12.59 \%, 11.39 \%$ and $17.13 \%$ respectively. The combined child prevalence of overweight/obesity in this study is consistent with individual studies conducted in different parts of Ethiopia $[12,18,39,40]$. The pooled prevalence of overweight/obesity of children in this study higher than $6.8 \%$ reported from 26 countries of Sub-Saharan Demographic and Health Surveys (DHSs) [49]. The finding in this study is also lower than the result from another 10-year systematic review and meta-regression result from Europe where $17.9 \%$ of 2-7 years-old children were either overweight or obese [50].

Prevalence of pooled overweight/obesity (11.39\%) among adolescents in this study is higher than individual studies in Jimma [34] and Addis Ababa $[21,32,35]$, but lower than other studies from Addis Ababa [14,20], Bahir dar [26], Hawassa [25], Diredawa
[13], and similar with another study conducted in Addis Ababa [23]. Regarding with adult overweight/obesity, the pooled result in this study $(14.69 \%)$ is lower than individual study from Hawassa (28.2\%) [24], and Dessie (28.5\%) [16], higher than finding from Arbaminch area of Ethiopia (10.8\%) [41], but in line with another study from Hawassa city (15.2\%) [19]. This variation in prevalence among different studies across different regions might be explained by differences in dietary habits, lifestyle factors; and climate and agro-ecology in individual studies while the combined prevalence is average of indicated studies.

In this study, the pooled effect size or odds ratio revealed that the study subjects (children, adolescents, and adults) from high socio-economic status were 1.89 times more likely to be either overweight/obese compared to the middle to low socioeconomic status households. This finding is in line with study evidence from further analysis of EDHS (2011) such that Ethiopian women from the richest households 1.8 times more likely to be overweighed/obese compared to their counterparts [38]. The finding also in line with another the result from systematic review and meta-analysis conducted in Ethiopia where children and women from high-income families were 3.16 times more likely to be overweight/obese children with middle and lowincome families [51]. On the contrary, the finding of the present study in disagreement with the study report 
a.

\begin{tabular}{|c|c|c|c|c|c|c|c|c|c|c|}
\hline \multirow{3}{*}{$\begin{array}{l}\text { Study or Subgroup } \\
\text { Berhane et.al., } 2020\end{array}$} & \multicolumn{2}{|c|}{ High SES/Income } & \multicolumn{3}{|c|}{ Low SES/Income } & \multirow{2}{*}{$\begin{array}{c}\text { Odds Ratio } \\
\text { M-H, Random, } 95 \% \mathrm{Cl}\end{array}$} & \multirow{2}{*}{\multicolumn{4}{|c|}{$\begin{array}{c}\text { Odds Ratio } \\
\text { M-H, Random, } 95 \% \mathrm{Cl}\end{array}$}} \\
\hline & \multirow{2}{*}{ Events } & \multirow{2}{*}{$\begin{array}{r}\text { Total } \\
625\end{array}$} & \multirow{2}{*}{$\begin{array}{r}\text { Events } \\
5\end{array}$} & \multirow{2}{*}{$\begin{array}{r}\text { Total } \\
203\end{array}$} & \multirow{2}{*}{$\begin{array}{r}\text { Weight } \\
9.8 \%\end{array}$} & & & & & \\
\hline & & & & & & \multicolumn{2}{|l|}{$5.48[2.19,13.75]$} & & \multirow{2}{*}{\multicolumn{2}{|c|}{$\longrightarrow$}} \\
\hline Birhanu et.al., 2019 & 60 & 451 & 8 & 139 & $11.6 \%$ & \multirow{2}{*}{\multicolumn{2}{|c|}{$\begin{array}{l}2.51[1.17,5.39] \\
4.97[2.95,8.39]\end{array}$}} & & & \\
\hline Mekonnen et.al., 2018 & 49 & 207 & 24 & 409 & $15.0 \%$ & & & & \multicolumn{2}{|l|}{$\longrightarrow$} \\
\hline Solomon et.al., 2016 & 139 & 709 & 395 & 2893 & $19.1 \%$ & \multicolumn{2}{|l|}{$1.54[1.25,1.91]$} & & \multicolumn{2}{|l|}{$\rightarrow$} \\
\hline Sorrie et.al., 2017 & 39 & 167 & 30 & 333 & $15.1 \%$ & \multicolumn{2}{|l|}{$3.08[1.83,5.17]$} & & $\longrightarrow$ & \\
\hline Tsedeke and Tefera, 2014 & 27 & 150 & 11 & 208 & $12.0 \%$ & \multicolumn{2}{|l|}{$3.93[1.88,8.21]$} & & \multirow{2}{*}{ 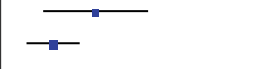 } & \\
\hline Weldearegay et.al., 2019 & 74 & 158 & 150 & 514 & $17.3 \%$ & $2.14[1.48,3.08]$ & & & & \\
\hline Total $(95 \% \mathrm{Cl})$ & & 2467 & & 4699 & $100.0 \%$ & \multirow[t]{2}{*}{$2.90[1.94,4.32]$} & & & $\infty$ & \\
\hline Total events & 464 & & 623 & & & & & & & \\
\hline \multicolumn{5}{|c|}{ Test for overall effect: $Z=5.21(P<0.00001)$} & Heterogeneity: Tau $^{2}=0.21 ;$ Chi $^{2}=27.95, d f=6(P<0.0001) ; I^{2}=79 \%$ & & 0.01 & $\begin{array}{c}0.1 \\
\text { Less } \mathrm{O} / 0 \text { in high SES/Inco }\end{array}$ & ${ }^{1} \stackrel{10}{10}$ & 100 \\
\hline
\end{tabular}

b.

\begin{tabular}{|c|c|c|c|c|c|c|c|c|c|c|c|}
\hline \multirow[b]{2}{*}{ Study or Subgroup } & \multicolumn{2}{|c|}{ High SES/Income } & \multicolumn{2}{|c|}{ Low SES/Income } & \multirow[b]{2}{*}{ Weight } & \multirow{2}{*}{$\begin{array}{l}\text { Odds Ratio } \\
\text { M-H, Random, } 95 \% \mathrm{Cl}\end{array}$} & \multirow{2}{*}{\multicolumn{5}{|c|}{$\begin{array}{l}\text { Odds Ratio } \\
\text { M-H, Random, } 95 \% \mathrm{Cl}\end{array}$}} \\
\hline & Events & Total & Events & Total & & & & & & & \\
\hline Anjulo et.al., 2017 & 25 & 49 & 78 & 275 & $8.0 \%$ & $2.63[1.42,4.88]$ & & & & & \\
\hline Askal et.al., 2015 & 76 & 625 & 5 & 203 & $6.7 \%$ & $5.48[2.19,13.75]$ & & & & & \\
\hline Desalew et.al., 2017 & 86 & 205 & 6 & 240 & $7.0 \%$ & $28.18[11.97,66.37]$ & & & & & \\
\hline Dessalegn et.al., 2017 & 15 & 119 & 52 & 481 & $8.0 \%$ & $1.19[0.64,2.20]$ & & & & & \\
\hline Dessalegn et.al., 2018 & 14 & 27 & 96 & 362 & $7.3 \%$ & $2.98[1.35,6.58]$ & & & & & \\
\hline Emana et.al., 2014 & 3 & 47 & 72 & 753 & $5.6 \%$ & $0.64[0.20,2.13]$ & & & & & \\
\hline Girmay and Hassen, 2018 & 134 & 830 & 8 & 120 & $7.5 \%$ & $2.70[1.29,5.65]$ & & & & & \\
\hline Kalkidan et.al., 2017 & 8 & 165 & 31 & 385 & $7.2 \%$ & $0.58[0.26,1.29]$ & & & & . & \\
\hline Mekonnen and Bogale, 2017 & 63 & 1212 & 38 & 1726 & $8.8 \%$ & $2.44[1.62,3.67]$ & & & & & \\
\hline Mulugeta et.al., 2016 & 26 & 89 & 42 & 357 & $8.3 \%$ & $3.10[1.77,5.41]$ & & & & & \\
\hline Tesfalem et.al., 2013 & 25 & 141 & 71 & 554 & $8.5 \%$ & $1.47[0.89,2.41]$ & & & & 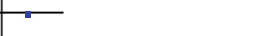 & \\
\hline Tsedey et.al., 2018 & 43 & 225 & 174 & 1021 & $8.9 \%$ & $1.15[0.79,1.67]$ & & & & - & \\
\hline Zelalem et.al., 2015 & 27 & 81 & 40 & 352 & $8.2 \%$ & $3.90[2.21,6.88]$ & & & & & \\
\hline Total $(95 \% \mathrm{Cl})$ & & 3815 & & 6829 & $100.0 \%$ & $2.36[1.52,3.65]$ & & & & & \\
\hline Total events & 545 & & 713 & & & & & & & & \\
\hline $\begin{array}{l}\text { Heterogeneity: } \operatorname{Tau}^{2}=0.52 ; \mathrm{Ch} \\
\text { Test for overall effect: } Z=3.83\end{array}$ & $\begin{array}{l}=78.56, d f \\
P=0.0001)\end{array}$ & $12(P<$ & $0.00001) ; 1$ & $85 \%$ & & & 0.01 & $\begin{array}{c}0.1 \\
\text { Less } \mathrm{O} / 0 \text { in } \mathrm{hi}\end{array}$ & SES/Inco & High O/O in high SES/Inco & $\overrightarrow{100}$ \\
\hline
\end{tabular}

C.

\begin{tabular}{|c|c|c|c|c|c|c|c|c|c|c|}
\hline \multirow[b]{2}{*}{ Study or Subgroup } & \multicolumn{2}{|c|}{ High SES/Income } & \multicolumn{2}{|c|}{ Low SES/Income } & \multirow[b]{2}{*}{ Weight } & \multirow{2}{*}{$\begin{array}{c}\text { Odds Ratio } \\
\text { M-H, Random, } 95 \% \mathrm{Cl}\end{array}$} & \multirow{2}{*}{\multicolumn{4}{|c|}{$\begin{array}{c}\text { Odds Ratio } \\
\text { M-H, Random, } 95 \% \mathrm{Cl}\end{array}$}} \\
\hline & Events & Total & Events & Total & & & & & & \\
\hline Bogale and Zewale, 2019 & 50 & 129 & 80 & 316 & $17.1 \%$ & $1.87[1.21,2.89]$ & & & \multicolumn{2}{|l|}{$\longrightarrow$} \\
\hline Darebo et.al., 2019 & 50 & 109 & 98 & 442 & $17.0 \%$ & $2.97[1.92,4.61]$ & & & $\longrightarrow$ & \\
\hline Gutema et.al., 2020 & 131 & 1006 & 227 & 2340 & $27.4 \%$ & $1.39[1.11,1.75]$ & & & \multirow[t]{2}{*}{$\rightarrow$} & \\
\hline Nega and Beyene, 2019 & 9 & 22 & 42 & 184 & $6.2 \%$ & \multirow{2}{*}{$\begin{array}{l}2.34[0.94,5.86] \\
1.98[1.163 .391\end{array}$} & & & & \\
\hline Samuel et.al., 2019 & 26 & 61 & 167 & 612 & $13.5 \%$ & & & & $\longrightarrow$ & \\
\hline Yosef et.al., 2020 & 125 & 200 & 99 & 200 & $18.7 \%$ & $1.70[1.14,2.53]$ & & & - & \\
\hline Total $(95 \% \mathrm{Cl})$ & & \multirow[t]{2}{*}{1527} & & 4094 & $100.0 \%$ & \multirow[t]{2}{*}{$1.87[1.46,2.41]$} & & & $>$ & \\
\hline Total events & 391 & & 713 & & & & & & & \\
\hline \multicolumn{7}{|c|}{ Heterogeneity: $\operatorname{Tau}^{2}=0.05 ; \mathrm{Chi}^{2}=10.08, \mathrm{df}=5(P=0.07) ; 1^{2}=50 \%$} & $\stackrel{\vdash}{\circ}$ & 0.1 & & 100 \\
\hline \multicolumn{5}{|c|}{ Test for overall effect: $Z=4.91(P<0.00001)$} & & & & Less $0 / 0$ in high SES/Inco & High $\mathrm{O} / \mathrm{O}$ in hi & igh SES/Inco \\
\hline
\end{tabular}

Figure 3: Forest plot for the pooled odds ratio of the association between socio-economic status and overweight/obesity in Ethiopia, 2020: -a: Children; b: Adolescents; c: Adults.

of further analysis of the Ethiopian demographic and health survey (EDHS) 2016 where the richest quintiles have no association with childhood overweight/obesity in Ethiopia [52]. The result of increased overweight/ obesity with high socio-economic status is also in line with other studies $[53,54]$. Another systematic review conducted in developing countries documented similar findings where obesity is a problem of the rich in lowincome countries for both men and women, while there is a mixed picture in middle-income countries [55]. On the contrary, a survey of household data from 103 countries by Templin, et al. (2019), documented that overweight prevalence increased substantially among the poorest and stayed mostly unchanged among the wealthiest [56]. Similarly, another meta-analysis by Kim and von dem Knesebeck (2018), revealed that lowerincome is associated with subsequent obesity compared to higher-income [57].

This positive correlation might be, even though the overweight/obesity is a complex, multifactorial condition affected by genetic and non-genetic factors $[58,59]$, and high socio-economic status could influence energy consumption and expenditure [60]. The imbalance increment of energy intake more than energy expenditure is considered the fundamental cause of obesity and overweight [61]. Besides, the wealthiest 
households would be able to afford and demand surplus foods and might be less likely to engage manual intensive work leading to less energy expenditure. On the contrary, low-income households might experience food scarcity and moderate to low food intake. Moreover, peoples from low to medium SES tend to be engaged in labor work that requires higher energy expenditure concomitantly losing body fat storage [62]. The negative correlation of low SES with overweight/ obesity might be explained by that low/middle-income households probably less likely to afford expensive, nutritious, nutrient-dense, and low-calorie food but focusing in high calorie, less nutritious and cheap priced food. This truth was documented by scholars such that low-calorie food will likely be expensive for the poor leading to the consumption of a more energy-dense $\operatorname{diet}[63,64]$.

It is well documented that obesity is a risk factor for non-communicable diseases (NCD). At the same time, decreases quality life increases health care costs and absenteeism. Costs become unmanageable by health budget in poor countries and aligned health initiatives (more toward communicable diseases [65]. Since Ethiopia is one of the low-income countries, the current finding implies Ethiopia would be fall under the triple burden of malnutrition (micronutrient deficiency, undernutrition and over nutrition) leading to further non-communicable disease initiatives.

\section{Conclusions}

The current meta-analysis result revealed that the pooled prevalence of overweight/obesity among children, adolescents, and adults is considerably high. This indicates that overweight and or obesity is becoming an emerging nutrition-related problem. High socioeconomic status is significantly positively associated with childhood, adolescents, and adults in Ethiopia.

\section{Recommendations}

Since overweight/obesity is significantly high among children, adolescents and adults, the government of Ethiopia, health care providers, non-governmental organizations and private institutions working on childhood, adolescents and adult health should give emphasize on prevention and control of overweight and or obesity. Intensive nutrition education and counseling should be provided for households of high socioeconomic status to focus on less energy-dense or high nutrient-dense foods and engage in physical activities. Policies that focus on prevention and control of overweight/obesity with special emphasis of wealthy households should be designed and implemented in Ethiopia.

\section{Strengths and Limitations of the Study}

The main strength of this systematic review and meta-analysis is that studies from different regions of Ethiopia generated the aggregate status of overweight and obesity. The study utilized articles from reputable multiple databases. The study also generated clear information on wealth status and overweight/obesity by narrowing the confusion.

\section{Limitation of the Study}

The study focused only on published articles. Other grey literature possibly missed from the study. There might be many unpublished studies in different higher institutions of Ethiopia though it could be accessed. All the steps were conducted by one reviewer only.

\section{Declarations}

\section{Ethics approval and consent to participate}

Not applicable.

\section{Consent for publication}

Not applicable.

\section{Availability of data and material}

The datasets used and analyzed for this study will be available upon the request of the corresponding author.

\section{Competing interests}

The author declares that I have no competing interests.

\section{Funding}

No fund is obtained for this study.

\section{Authors' Contributions}

TZ (the author): Conception of research protocol, study design, literature review, data extraction, quality assessment, data analysis, interpretation and writing manuscript.

\section{Acknowledgement}

I would gratefully thank all the authors of original articles used in this study. I would also like to thank all staffs from hawassa human nutrition departments for they support on quality assessment.

\section{References}

1. Organization WH (2014) Obesity and overweight's. Fact sheet No. 311.

2. Center for Disease Control and Prevention (2015) Adolescent and school health, Childhood obesity facts.

3. UNICEF/World Health Organization (WHO)/World Bank Group (2017) Joint child malnutrition estimates.

4. NCD Risk Factor Collaboration 2018.

5. FAO I, UNICEF, WFP and WHO (2019) The state of food security and nutrition in the world 2019. Safeguarding against economic slowdowns and downturns. Rome, FAO. Licence: CC BY-NC-SA 3.0 IGO.

6. CSA and ICF: Central Statistical Agency (CSA) [Ethiopia] 
and ICF (2016) Ethiopia demographic and health survey 2016: Key indicators report. Addis Ababa, Ethiopia, and Rockville, Maryland, USA.

7. CSA and ICF: Central Statistical Agency (CSA) [Ethiopia] and ICF (2012) Ethiopia demographic and health survey 2011: Key indicators report. Addis Ababa, Ethiopia, and Rockville, Maryland, USA.

8. UNICEF: Annual report 2016, Ethiopia.

9. WHO: WHA global nutrition targets 2025: Childhood overweight policy brief. Geneva, Switzerland: World Health Organization.

10. Visscher TLS, Seidell JC (2001) The public health impact of obesity. Annu Rev Public Health 22: 355-375.

11. Taylor VH, Forhan M, Vigod SN, Mclntyre RS, Morrison KM (2013) The impact of obesity on quality of life Best Pract Res Clin Endocrinol Metab 27: 139-146.

12. Sorrie MB, Yesuf ME, Michael TGGG (2017) Overweight/ obesity and associated factors among preschool children in Gondar City, Northwest Ethiopia: A cross-sectional study. PLoS One 12: e0182511.

13. Dessalew A, Mandesh A, Semahegn A (2017) Childhood overweight, obesity and associated factors among primary schoolchildren in dire dawa, eastern Ethiopia; a crosssectional study. BMC Obesity 4: 20.

14. Dereje D, Yirgu R, Chichiabellu TY (2018) Magnitude of overweight/obesity and associated factors among high school adolescents' in Addis Ababa, Ethiopia. Journal of Nutritional Disorders \& Therapy 8: 2.

15. Yohannes M (2019) Prevalence of overweight and obesity among office-based urban civil servants in southern nations, nationalities and peoples' region, Ethiopia. Ethiop Med J 57.

16. Dagne S, Gelaw YA, Abebe Z, Wassie MM (2019) Factors associated with overweight and obesity among adults in northeast Ethiopia: A cross-sectional study. Diabetes Metab Syndr Obes 12: 391-399.

17. Weldearegay HG, Gebrehiwot TG, Abrha MW, Mulugeta A (2019) Overweight and obesity among children under five in Ethiopia: Further analysis of 2016 national demographic health survey: A case control study. BMC Res Notes 12 716

18. Taye K (2019) Level of overweight, obesity and associated factors among preschool children in dire dawa city, eastern Ethiopia, 2016. Journal of Medicine, Physiology and Biophysics 52.

19. Bogale KA, Zewale TA (2019) Determinant factors of overweight/obesity among federal ministry civil servantsin Addis Ababa, Ethiopia: A call for sectorwise occupational health program. BMC Research Notes 12: 449.

20. Moges T, Gebremichael B, Shiferaw S, Yirgu R (2018) Is inadequate play area in schools associated with overweight among students in Addis Ababa, Ethiopia? A comparative cross-sectional study. Epidemiology and Health 40: e2018017.

21. Alemu E, Atnafu A, Yitayal M, Yimam K (2014) Prevalence of overweight and/or obesity and associated factors among high school adolescents in Arada sub city, Addis Ababa, Ethiopia. Journal of Nutrition \& Food Sciences 4.

22. Girmay AM, Hassen MN (2018) Prevalence of overweight and obesity and associated factors among private primary school students in gulele sub-city of Addis Ababa, Ethiopia. Epidemiology (Sunnyvale) 8: 352.
23. Shegaze M, Wondafrash M, Alemayehu AA, Mohammed S, Shewangezaw Z, et al. (2016) Magnitude and determinants of overweight and obesity among high school adolescents in Addis Ababa, Ethiopia. International Journal of Medical and Health Sciences 10.

24. Darebo T, Mesfin A, Gebremedhin S (2019) Prevalence and factors associated with overweight and obesity among adults in Hawassa city, southern Ethiopia: Acommunity based cross-sectional study. BMC Obesity 6: 8.

25. Teshome T, Singh P, Moges D (2013) Prevalence and associated factors of overweight and obesity among high school adolescents in urban communities of Hawassa, southern Ethiopia. Current Research in Nutrition and Food Science 1: 23-36.

26. Anteneh ZA, Gedefaw M, Tekletsadek KN, Tsegaye M, Alemu D (2015) Risk factors of overweight and obesity among high school students in Bahir Dar City, North West Ethiopia: School based cross-sectional study. Advances in Preventive Medicine 2015.

27. Bereket AH, Beyero M, Fikadu AR, Bosha T (2017) Risk factors for overweight and obesity in private high school adolescents in Hawassa City, Southern Ethiopia: A Casecontrol Study. Food and Public Health 7: 29-34.

28. Review Manager (RevMan) (2014) Version 5.3. Copenhagen: the nordic cochrane centre, the cochrane collaboration.

29. Rücker G, Schwarzer G, Carpenter J, Schumacher M (2008) Undue reliance on 12 in assessing heterogeneity may mislead. BMC Med Res Methodol 8: 79.

30. Higgins J, Thompson S (2002) Quantifying heterogeneity in a metaanalysis. Stat Med 21: 1539-1558.

31. Egger M, Smith GD, Schneider M, Minder C (1997) Bias in meta-analysis detected by a simple, graphical test. BMJ Open 315: 629-634.

32. Berbada DA, Haidar J, Gebremichael G, Haftu D (2017) Magnitude of double burden of malnutrition and its associated factors among selected in-school adolescents: Evidence from South Ethiopia. International Journal of Nutrition and Metabolism 9: 30-37.

33. Nega G, Endris M, Teferi D, Nana A, Michael RW (2015) Dietary diversity and associated factors among rural households in south Gondar zone, Northwest Ethiopia.

34. Hassen K, Gizaw G, Belachew T (2017) Dual burden of malnutrition among adolescents of smallholder coffee farming households of jimma zone, Southwest Ethiopia. Food and Nutrition Bulletin 38: 196-208.

35. Askal T, Yifokr T, Bekri M, Getachew TG (2015) Prevalence and associated factors of overweight and/or obesity among primary school children in bole sub-city, Addis Ababa, Ethiopia. Journal of Nutrition \& Food Sciences 5: 409.

36. Berhane $H Y$, Jirström M, Abdelmenan $S$, Berhane $Y$, Alsanius B, et al. (2020) Social stratification, diet diversity and malnutrition among preschoolers: A survey of Addis Ababa, Ethiopia. Nutrients 12: 712.

37. Gali N, Tamiru D, Tamrat M (2017) The emerging nutritional problems of school adolescents: Overweight/obesity and associated factors in Jimma Town, Ethiopia. Journal of Pediateric Nursing 35: 98-104.

38. Abrha S, Shiferaw S, Ahmed KY (2016) Overweight and obesity and its sociodemographic correlates among urban Ethiopian women: Evidence from the 2011 EDHS. BMC Public Health 16: 636. 
39. Mekonnen T, Tariku A, Abebe SM (2018) Overweight/ obesity among school aged children in Bahir Dar City: Cross sectional study. Italian Journal of Pediatrics 44: 17.

40. Wolde T, Belachew T (2014) Prevalence and determinant factors of overweight and obesity among preschool children living in Hawassa city, South Ethiopia. Food Science and Quality Management 29.

41. Gutema BT, Chuka A, Kondale M, Ayele G, Kote M, et al. (2020) The burden of malnutrition among adults residing in arba minch health and demographic surveillance site (HDSS): A WHO STEPS Survey. Journal of Nutrition and Metabolism 2020.

42. Ejara B, Taye K, Tiki T (2019) Level of overweight, obesity and associated factors among preschool children in dire dawa city, Eastern Ethiopia, 2016. Journal of Medicine, Physiology and Biophysics 52.

43. Yosef T, Bogale B, Destaw A, Weldu A (2020) The burden of overweight and obesity among long-distance truckers in Ethiopia. Hindawi Journal of Obesity 2020.

44. WHO [World Health Organization] (2016) Consideration of the evidence on childhood obesity for the commission on ending childhood obesity: Report of the Ad hoc working group on science and evidence for ending childhood obesity, Geneva, Switzerland.

45. Lobstein T, Baur L, Uauy R, IASO International Obesity Task Force (2004) Obesity in children and young people: A crisis in public health. Obes Rev 1: 4-104.

46. Engeland A, Bjørge T, Søgaard AJ, Tverdal A (2003) Body mass index in adolescence in relation to total mortality: 32year follow-up of 227,000 Norwegian boys and girls. Am J Epidemiol 157: 517-523.

47. Neupane S, Prakash K, Doku DT (2016) Overweight and obesity among women: Analysis of demographic and health survey data from 32 sub-Saharan African countries. BMC Public Health 16: 30.

48. Yu Z, Han S, Chu J, Xu Z, Zhu C, et al. (2012) Trends in overweight and obesity among children and adolescents in china from 1981 to 2010: A meta-analysis. PLoS One 7: e51949.

49. Gebremedhin S (2015) Prevalence and differentials of overweight and obesity in preschool children in SubSaharan Africa. BMJ Open 5: e009005.

50. Miguel MG, Oliveira A, Redondo IC, Bueno CA, Carrascosa DPP (2019) Prevalence of overweight and obesity among European preschool children: A systematic review and meta-regression by food group consumption. Nutrients 11 : 1698.
51. Gebrie A, Alebel A, Zegeye A, Tesfaye B, Ferede A (2018) Prevalence and associated factors of overweight/ obesity among children and adolescents in Ethiopia: A systematic review and meta-analysis. BMC Obesity 5 .

52. Amare ZY, Ahmed ME, Meharie AB (2016) Determinants of nutritional status among children under age 5 in Ethiopia: Further analysis of the 2016 Ethiopia demographic and health survey. Global Health 15: 62.

53. Güngör NK (2014) Overweight and obesity in children and adolescents. J Clin Res Pediatr Endocrinol 6: 129-143.

54. Tanwi TS, Chakrabarty S, Hasanuzzaman S, Saltmarsh S, Winn S (2019) Socioeconomic correlates of overweight and obesity among ever-married urban women in Bangladesh. BMC Public Health 19.

55. Dinsa GD, Goryakin Y, Fumagalli E, Suhrcke M (2012) Obesity and socioeconomic status in developing countries: A systematic review. Obes Rev 13: 1067-1079.

56. Templin T, Hashiguchi TCO, Thomson B, Dieleman J, Bendavid E (2019) The overweight and obesity transition from the wealthy to the poor in low-and middle income countries: A survey of household data from 103 countries. . PLoS Medicine 16: e1002968.

57. Kim TJ, Knesebeck OVD (2018) Income and obesity: What is the direction of the relationship? A systematic review and meta-analysis. BMJ Open 8: e019862.

58. Han JC, Lawlor DA, Kimm SYS (2010) Childhood Obesity-2010: Progress and Challenges. Lacent 375: 1737-1748.

59. Lustig RH, Weiss R (2008) Disorders of energy balance. In: Sperling MA, Pediatric Endocrinology ( $3^{\text {rd }}$ edn), Saunders Elsevier, Philadelphia, 788-838.

60. Childhood obesity.

61. Ellulu M, Abed Y, Rahmat A, Ranneh Y, Ali F (2014) Global epidemic obesity. Epidemiology of obesity in developing countries: Challenges and prevention. Herbert Open Access Journal 2: 2.

62. World Health Organization (2003) Diet, nutrition and the prevention of chronic diseases. Geneva Report No: 916.

63. Drewnowski A, Specter SE (2004) Poverty and obesity: The role of energy density and energy costs. Am J Clin Nutr 79: 6-16.

64. Drewnowski A, Darmon N (2005) The economics of obesity: Dietary energy density and energy cost. Am J Clin Nutr 82: 265S-273S.

65. Thompson DL (2007) The costs of obesity: What occupational health nurses need to know. AAOHN J 55: 265-270. 